Piotr Szwed*

\title{
Application of Fuzzy Cognitive Maps to Analysis of Development Scenarios for Academic Units
}

\section{Introduction}

For many classes of problems it is difficult to make decisions, assessments or develop plans based on precise quantitative models. Fuzzy Cognitive Maps (FCM) are a wellknown tool for qualitative analysis of systems that uses a simple representation of knowledge in the form of a graph of concepts linked by causal relationships. The advantages of FCMs are the ease of gathering and representing knowledge and the simplicity of reasoning techniques, very close to neural networks.

This paper discusses the results of experiments aiming at application of FCMs to the analysis of development scenarios for research and teaching units (e.g. departments within faculties). The motivation for the work was an intention to investigate FCM techniques on a real, yet appealing example. The problem was chosen for two reasons. The first is a potential interest in the academic community in results of analyzes; the second is the ease of perception of the problem domain, what facilitates the interpretation of obtained results.

This paper is organized as follows: Section 2 gives a description of Fuzzy Cognitive Maps and related reasoning techniques, next Section 3 presents FCM model for the discussed problem, then Section 4 discusses the results of the analyzes for four key scenarios and finally Section 5 provides conclusions.

\section{Fuzzy Cognitive Maps}

Cognitive maps were first proposed by Axelrod [1] as a tool for modeling political decisions, then they were extended by Kosko [2, 3] by introducing fuzzy values. A large number of applications of FCM were reported, e.g. in project risk modeling, crisis management and decision making, the analysis of the development of economic systems, the introduction

* AGH University of Science and Technology, Krakow, Poland 
of new technologies [4], ecosystem analysis [5], signal processing and decision support in medicine. A survey on Fuzzy Cognitive Maps and their applications can be found in [6] and [7].

FCMs are directed graphs whose vertices represent concepts, whereas edges are used to express causal relations between them. A set of concepts $C=\left\{c_{1}, \ldots, c_{n}\right\}$ appearing in a model encompasses events, conditions or other relevant factors. System state is an $n$-dimensional vector of concept activation levels $(n=|C|)$. In contrast to condition/events Petri nets or state machines, where only two activation levels are used: 0 (inactive) and 1 (active), in FCM an activation level can be a real value belonging to $[0,1]$ or $[-1,1]$.

Causal relations between concepts are represented in FCM by edges and assigned weights. A positive weight of an edge linking two concepts $c_{i}$ and $c_{j}$ models a situation, where increase of the level of $c_{i}$ results in growing $c_{j}$; a negative weight is used to describe opposite rapport.

In the simplest FCM form, values from the set $\{1,0,-1\}$ are used as weights. They are graphically represented as a minus (-) sign attached to an edge, absence of edge or a plus (+) sign. While building FCM models, more fine-grained causal relations can be introduced. They are usually specified as linguistic values, e.g.: strong_negative, negative, medium_negative, neutral, medium_positive, positive $\}$, strong_positive, and in a computational model mapped on values uniformly distributed over $[-1,1]$.

Causal relations between concepts in FCM can be represented by $n \times n$ influence matrix $\mathbf{E}=\left[e_{i j}\right]$, whose elements $e_{i j}$ are weights assigned to edges linking $c_{j}$ and $c_{i}$ or have 0 values, if there is no link between them.

Figure 1 gives an example of FCM graph, whose vertices were assigned with concepts $c_{1}, c_{2}, c_{3}$ and $c_{4}$, whereas edges with linguistic influence weights. The corresponding $\mathbf{E}$ matrix is given by the formula (1). The selection of values corresponding to linguistic values is arbitrary, in this case values: $-1,-0.66,-0.33,0,0.33,0.66,1$ were taken.

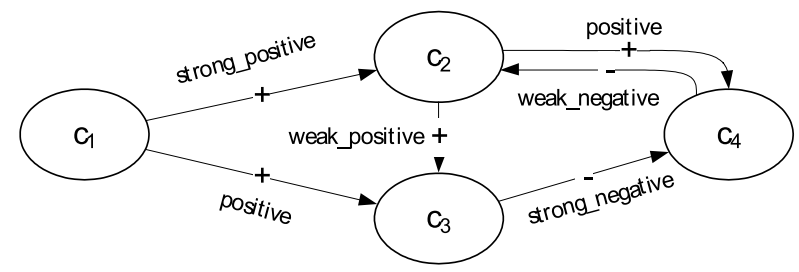

Fig. 1. Example of FCM graph. Vertices are assigned with concepts, directed edges with linguistic influence weights

$$
E=\left[\begin{array}{cccc}
0 & 0 & 0 & 0 \\
1 & 0 & 0 & -0.33 \\
0.66 & 0.33 & 0 & 0 \\
0 & 0.66 & -1 & 0
\end{array}\right]
$$




\subsection{Reasoning with Fuzzy Cognitive Maps}

Reasoning with FCM consist in building a sequence of states: $\alpha=A(0), A(1), \ldots, A(k), \ldots$ starting form an initial vector of activation levels of concepts. Consecutive elements are calculated according to the formula (2). In the $k+1$ iteration the vector $A(k)$ is multiplied by the influence matrix $\mathbf{E}$, then the resulting activation levels of concepts are mapped onto the assumed range by means of an activation function (called also a splashing function).

$$
A_{i}(k+1)=S_{i}\left(\sum_{j=1}^{n} e_{i j} A_{j}(k)\right)
$$

The selection of an activation function depends on assumptions regarding the model, in particular selected range of activation levels and the decision for using continuous or discrete values. Multiplication of $n$-dimensional square matrix $\mathbf{E}$ and a vector, both containing elements, whose absolute values are bounded by 1 , results in a vector having elements in $[-n, n]$. Values from this interval should be mapped by an activation function into the range $[-1,1]$ (or $[0,1])$ preserving monotonicity and satisfying $S(0)=0$ (or $S(0)=0.5$ in the second case).

In the further analysis three activation functions were used:

$$
\begin{aligned}
& S_{\text {cut }}(x)= \begin{cases}1, & \text { dla } x>1 \\
x, & \text { dla } x \geq-1 \wedge x \leq 1 \\
-1, & \text { dla } x<-1\end{cases} \\
& S_{\text {exp }}(x)= \begin{cases}1-e^{-m x}, & \text { dla } x \geq 0 \\
-1+e^{m x} x, & \text { dla } x<0\end{cases} \\
& S_{\operatorname{logmod}}(x)=\frac{1}{1+e^{-m x}}
\end{aligned}
$$

Function $S_{\text {cut }}(x)$ maps arguments onto the interval $[-1,1]$ replacing values laying outside by the lower or upper bound. Function $S_{\text {exp }}(x)$ has similar shape to do $S_{\text {cut }}(x)$, but more smoothed and flattened, what is controlled by the coefficient $m$ typically having a value ranging from 1 to 5. Function $S_{\text {logmod }}(x)$ is very often referenced in the FCM bibliography, e.g. $[6,7,8]$. Its range is $[0,1]$, and it maps 0 to 0.5 . The logmod function seems to be the most consistent with a classical fuzzy approach, because its range corresponds to the range of membership functions of fuzzy sets. 
It should be mentioned then in the FCM bibliography, e.g. [7] another form of the equation (2) can be found:

$$
A_{i}(k+1)=S_{i}\left(\sum_{\substack{j=1 \\ j \neq i}}^{n} e_{i j} A_{j}(k)+A_{i}(k)\right)
$$

Formula (6) explicitly states, that changes of activation levels for a concept $c_{i}$ calculated in the $k$-th iteration accumulate with the previous value $A_{i}(k)$. It corresponds to an implicit assumption, that coefficients $e_{i i}$ of the influence matrix are equal to 1 . In a general case, it need not to be satisfied, therefore during analyzes equation (2) was used, as more general and flexible.

Basically, a sequence of consecutive states $\alpha=A(0), A(1), \ldots, A(k), \ldots$ is infinite. However, it was shown that after $k$ iterations, where $k$ is a number close to the rank of matrix $\mathbf{E}$ a steady state is reached or a cycle occurs. (This observation is not surprising, as analogous to properties of Markov chains.)

Hence, a stop criterion for the reasoning algorithm in the \$ $\$$ step:

$$
\exists j<k: d(A(k), A(j))<\varepsilon
$$

where $d$ is a distance and $\varepsilon$ a small value, e.g. $10^{-2}$.

A sequence of states $\alpha$ can be can be interpreted in two ways:

1) It can be treated as a representation of a dynamic behavior of modeled system. In this case there exist implicit temporal relations between consecutive system states, and the whole sequence describes an evolution of the system in the form of a scenario.

2) As non-monotonic fuzzy inference process, in which selected elements of a steady state are interpreted as reasoning results. An occurrence of a cycle can be treated as a form of undecidability.

In this paper we focus on the first approach.

\subsection{Scenario analysis}

Concept building up an FCM can be divided into two sets: $C_{\mathrm{S}}$ comprised of state variables and $C_{\mathrm{D}}$ grouping decisions. Initial activation levels for state variables in $C_{\mathrm{S}}$ result from assessment of the current situation. In the course of reasoning they evolve under constant values of decision variables. Hence, an initial state vector $A(0)$ determines a scenario of system behavior that, after a number of iterations, leads to a stable vector of state variables or to a cycle.

FCM can be applied to scenario analysis in two manners. The first aims at selecting decisions that for a given initial state (e.g. company condition) lead to the desired goal. 
For this purpose, various combinations of decisions and their influence on development scenarios are examined. The second consists in determining, how a set of constant decisions affects systems varying in initial states. An example of application can be an analysis of influence of new regulations on groups of enterprises in similar standing. In this work the second approach was taken: we analyze scenarios for the development of academic units varying in initial conditions under the assumption of constant influences of decisions related to current law regulations.

\section{FCM Model}

The FCM model for research and teaching units was comprised of 10 concepts: Parametric evaluation, Papers, Grants, Teaching workload, Ministry funding, Professors, Assistant professors, Assistants, Law and Students. It should be observed that these concepts are not numeric values, although, in the calculation model they are assigned with values from the normalized membership range. They have also a vague semantics, e.g. the concept Assistant professors can be perceived as "potential of assistant professors", what can be interpreted both as their number and as a research or teaching quality. This potential can grow by increasing the number of assistant professors, but also by increasing the research effectiveness of the whole group or selected individuals.

Figure 2 shows the influence matrix $\mathbf{E}$ for the FCM model. Values of its elements correspond to assumed linguistic terms describing influences: strong_negative (-1), negative (-0.66), weak_negative $(-0.33)$, neutral $(0)$, weak_positive $(0,33)$, positive $(0.66)$ and strong_positive (1). Below a justification for particular weights is given.

1) Parametric evaluation. Its outcome depends on Papers and Grants, with positive weights 0.66 and 0.33 respectively.

2) Papers. Positive influence have Grants (0.66), Ministry funding (0.33) and research staff, as publication producers; negative influence $(-0.66)$ has Teaching workload.

3) Grants. Positive influence (0.66) have Papers (they increase the probability of being awarded with a grant) and the availability and involvement of performers (staff and students); negative influence has Teaching workload $(-0.66)$.

4) Teaching workload. Positive influence (1.0) have Students, whereas it diminishes with growing staff ( -0.33 weights are assigned to various staff members).

5) Ministry funding. Depends on the outcome of Parametric evaluation, staff and number of Students (0.33).

6) Professors. The model takes into account several factors influencing the potential of professors: Parametric evaluation - weak result may cause migration of professors (0.33). The highest influence is attributed to Papers (0.66), because to get a degree appropriate research achievements should be gathered. Influence factors (0.33) were given to Assistant professors, Assistant and Students. Statistically, in a large group of Assistant professors, more individuals striving for habilitation can be found. 
Their efforts can be supported by collaboration with assistants and students. Positive influence ( 0.33 ) on the number of professors has Law regulations (limited period of employment as an assistant professor).

7) Assistant professors. Their number is influenced by: Grants (0.33), and Teaching workload (0.33) - a lack of performers or a workload can be a source of a pressure on employment, available Ministry funding (0.66) and the supply of Assistants (0.33). Law is the factor that negatively influences the number of assistant professors $(-0.66)$, because imposed limits of the employment period will stimulate departures of employees, but also encourage to leave the university after obtaining Ph.D. degree.

8) Assistants. In the case of assistants influences of Grants, Teaching workload and Ministry funding are the same, as for Assistant professors, respectively:0.33, 0.33 and 0.66. The number of assistants is positively influenced by Professors (looking for Ph.D. students) and the supply of Students (0.33 i 0.33 ).

9) Law is a constant decision variable, it is not influenced by any factor in the considered model.

10) Students. Small positive influence (0.33) on potential of students have Professors and Assistant professors by their involvement in the modernization of teaching curricula.

The above list does not comprise values of the weights placed at the diagonal of $\mathbf{E}$ matrix, i.e. factors responsible for the decay of values resulting from previous iterations. It was assumed that for Parametric evaluation Papers and Students these coefficients have values $0.33,0.66$ and 0.66 respectively. For each type of staff members they are set to 1 , thus their previous values will cumulate with changes. The weight assigned to Law is also equal to 1 , as its value should be constant throughout scenarios.

Parametric evaluation

Papers

Grants

Teaching workload

Ministry funding

Professors

Assistant professors

Assistants

Law

Students

\begin{tabular}{|c|c|c|c|c|c|c|c|c|c|}
\hline 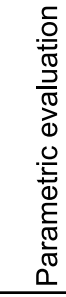 & $\begin{array}{l}\stackrel{\infty}{\Phi} \\
\stackrel{2}{\sigma} \\
0\end{array}$ & $\begin{array}{l}\frac{\infty}{\pi} \\
\frac{\pi}{\pi} \\
0\end{array}$ & 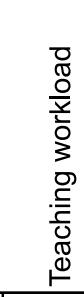 & 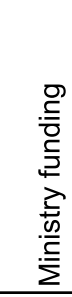 & 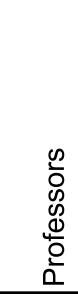 & 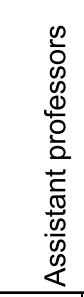 & $\begin{array}{l}\frac{0}{1} \\
\frac{\pi}{0} \\
\frac{.0}{5} \\
\frac{0}{4}\end{array}$ & 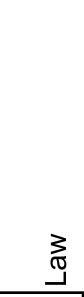 & $\begin{array}{l}\frac{0}{0} \\
\frac{1}{0} \\
\frac{0}{2} \\
\stackrel{0}{0}\end{array}$ \\
\hline 0.33 & 0.66 & 0.33 & 0 & 0 & 0 & 0 & 0 & 0 & 0 \\
\hline 0 & 0.66 & 0.66 & -0.66 & 0.33 & 0.66 & 0.33 & 0.33 & 0 & 0 \\
\hline 0 & 0.66 & 0.33 & -0.66 & 0 & 0.33 & 0.33 & 0.33 & 0 & 0.33 \\
\hline 0 & 0 & 0 & 0 & 0 & -0.33 & -0.33 & -0.33 & 0 & 1 \\
\hline 0.33 & 0 & 0 & 0 & 0 & 0.33 & 0.33 & 0.33 & 0 & 0.33 \\
\hline 0.33 & 0.66 & 0 & 0 & 0 & 1 & 0.33 & 0.33 & 0.33 & 0.33 \\
\hline 0 & 0 & 0.33 & 0.33 & 0.66 & 0 & 1 & 0.33 & -0.66 & 0 \\
\hline 0 & 0 & 0.33 & 0.66 & 0.66 & 0.33 & 0 & 1 & 0 & 0.33 \\
\hline 0 & 0 & 0 & 0 & 0 & 0 & 0 & 0 & 1 & 0 \\
\hline 0 & 0 & 0 & 0 & 0 & 0.33 & 0.33 & 0 & 0 & 0.66 \\
\hline
\end{tabular}

Fig. 2. Influence matrix 


\section{Scenario analyzes}

After building the FCM model a few dozen of scenarios were analyzed for activation functions defined by formulas (3), (4) and (5). It should be observed that, in spite of very promising benchmarks in literature [8], the application of logmod function $\left(S_{\text {logmod }}\right)$ did not yield valuable results, because obtained scenarios were hard to differentiate. The representation of system behaviors for activation functions $S_{\text {cut }}$ and $S_{\text {exp }}$ were very similar, therefore they will be alternately used in results discussion.

The rest of the section presents typical development scenarios for the four most salient cases: a strong academic unit, a unit in a medium standing, a weak unit having a certain potential of development and just a weak unit.

\subsection{Scenario 1: strong unit}

The initial values of the state vector for this scenario are the following: [ Parametric evaluation $=1$, Papers $=1$, Grants $=1$, Teaching workload $=1$, Ministry funding $=1$, Professors $=1$, Assistant professors $=1$, Assistants $=1$, Law $=1$, Students $=1]$. Figure 3 presents the development of state variables in consecutive 10 iterations leading to a steady state. A number of simulations for slightly varying parameters was also analyzed. The scenario is insensitive to changes of staff potential, as well as the number of students.

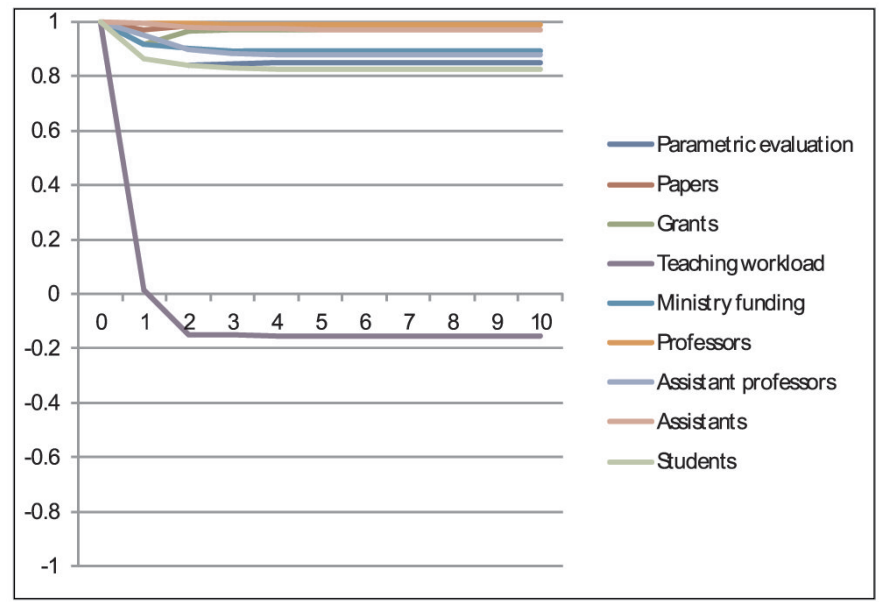

Fig. 3. Evolution of state variables for scenario $1 ; S_{\text {exp }}$ activation function was used

\subsection{Scenario 2: unit in a medium standing}

For a unit in a medium standing the following initial values of state vector were assumed: $[$ Parametric evaluation $=0$, Papers $=0$, Grants $=0$, Teaching workload $=0.7$, 
Ministry funding $=0$, Professors $=0.1$, Assistant professors $=0$, Assistants $=0$, Law $=1$, Students $=0.7]$. The graphs shown in Figure 4 obtained by utilizing application functions $S_{c u t}$ and $S_{\text {exp }}$ are qualitatively very similar.

a)

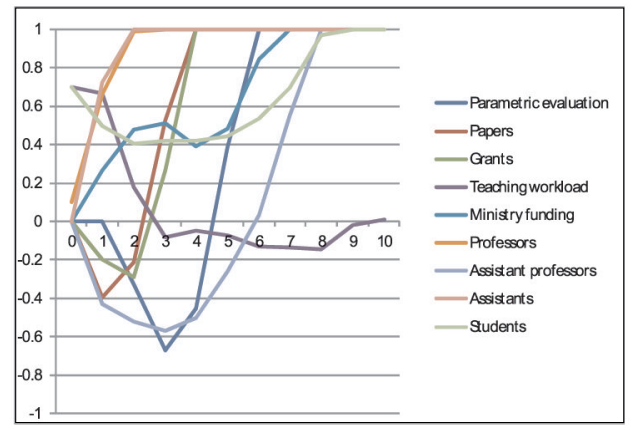

b)

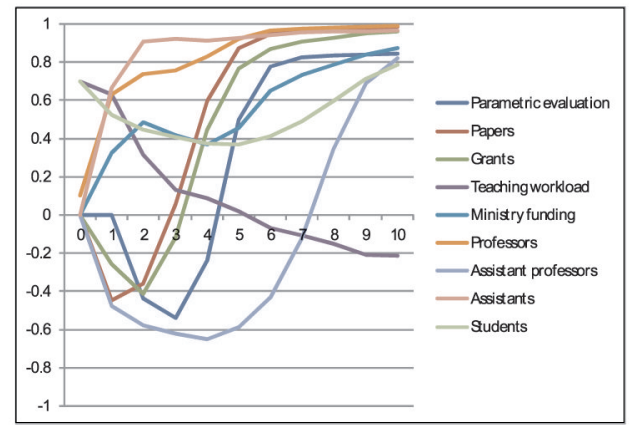

Fig. 4. Evolution of state variables for a unit in medium standing for activation function $S_{\text {cut }}$ (a) and $S_{\exp }$ (b)

The scenario is sensitive to the initial activation level of Students. The level 0 is a boundary value. For the case of $S_{\text {cut }}$ activation function after 10 iterations the steady state vector is reached $[-1,-1,-1,-0.01,-1,-1,-1,-1,1,-1]$, whereas for $S_{\text {exp }}$ the vector $[0.84,0.98,0.92,-0.49,0.64,0.98,-0.51,0.89,1,0.14]$ that can be perceived as a good forecast. Moreover, the result is practically independent (qualitatively) of $m$ constant in formula (4). An estimation of student potential is for this scenario a sensitive point. Decreasing the initial value to -0.3 causes that also for $S_{\exp }$ function a negative forecast is observed.

\subsection{Scenario 3: a weak unit with a potential}

An interesting case is the course of scenario (Fig. 5), for units that can be characterized as weak, although having a development potential. The potential is associated with staff members and students. The following initial values of the state vector are representative for the case: [Parametric evaluation $=-0.3$, Papers $=-0.3$, Grants $=-0.7$, Teaching workload $=0.7$, Ministry funding $=0$, Professors $=0.3$, Assistant professors $=0.2$, Assistants $=0.2$, Law $=1$, Students $=0.7]$.

For such unit FCM based reasoning yields positive development scenarios (in a longer horizon). Results for both activation functions are amazingly consistent. In both cases, the evolution directions are sensitive to the level of initial potential. Decreasing of any initial value by 0.2 for Professors, Assistant professors, Assistants or Students leads to a negative forecast. 
a)

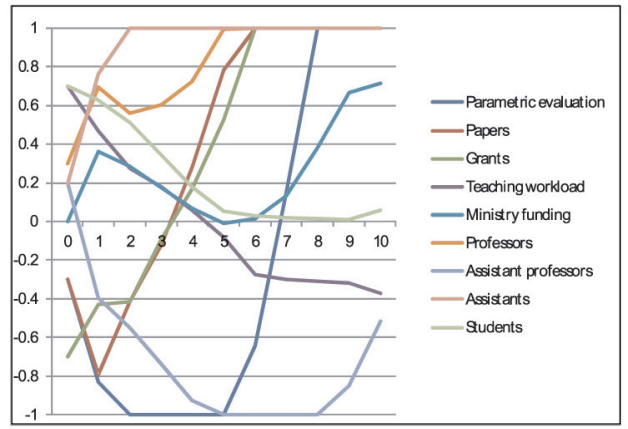

b)

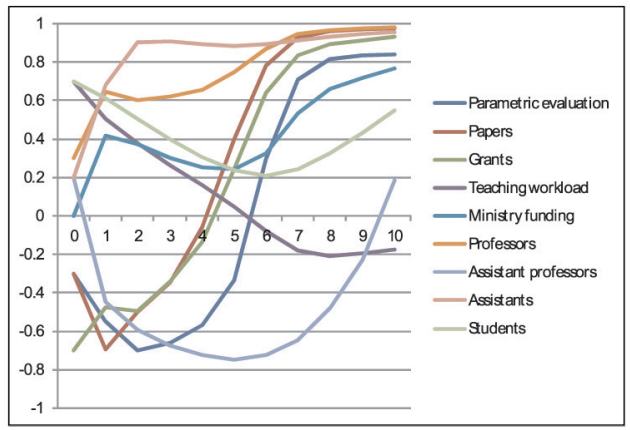

Fig. 5. Evolution of state variables for scenario 3 for activation functions (a) $S_{\text {cut }}$ and (b) $S_{\text {exp }}$

\subsection{Scenario 4: a weak unit}

The last discussed example is a weak unit, which has balanced (adjusted to current needs) level of staff focused on teaching. Initial values of the state vector are: [Parametric evaluation $=-0.5$, Papers $=-0.3$, Grants $=-0.7$, Teaching workload $=0.7$, Ministry fund ing $=0$, Professors $=0$, Assistant professors $=0$, Assistants $=0$, Law $=1$, Students $=0.7]$. Figure 6 shows a typical course of states. An interesting behavior, repeating in several simulations for slightly changed parameters, is forecasted an increase of Assistants in a few starting iterations.

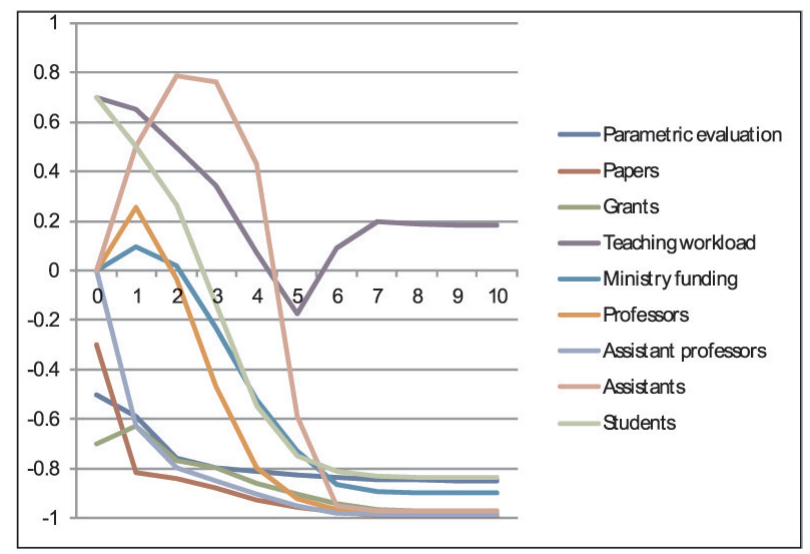

Fig. 6. Evolution of state variables for scenario $4 ; S_{\text {exp }}$ activation function was used

As it can be observed, the scenarios (1-3) in spite of high student potential, end with reduced Teaching workload. For the last scenario this parameter also diminishes, but is accompanied by a significant decrease of Students. 


\section{Conclusions}

In this paper we discuss an application of Fuzzy Cognitive Maps to analysis of development scenarios for academic units. For each scenario parallel calculations were conducted with three activation functions $S_{\text {cut }}, S_{\text {exp }}$ i $S_{\text {logmod }}$ defined according to formulas (3), (4) and (5). In the latter case, ranges of activation levels were mapped on the interval [0,1]. The functions $S_{\text {cut }}$ and $S_{\text {exp }}$ yielded qualitatively similar results. In spite of promising benchmarks in the literature, the logmod function occurred unsuitable. Perhaps the reason should be sought in parameters of model, in particular a small number of negative influences. Indeed, the model was built with an implicit assumption, that concepts can be assigned with negative activation levels. Hence, a positive influence for a negative value of source may result in decreasing the target. An interesting alternative would be an application of complementary concepts and the removal of negative influences according to the technique proposed in [2].

Function $S_{\text {exp }}$ defined by the formula (4) has not been already reported. The experiments indicate that for models assuming the range of activation levels between -1 and 1 the function is a good alternative for cutting off activation functions, as it quickly leads to steady solutions, while ensuring more smooth shapes of curves.

An interesting problem is how to relate an iteration of reasoning to real time. Actually, the FCM model is not a quantitative dynamic model. In some cases, however, assigned weights are implicitly related to time. Values placed at the diagonal of the influence matrix are the examples, as they determine decay periods for activation levels. For Parametric evaluation the value 0.33 was assigned, what corresponds to a decay in 3 iterations; the coefficient selected for Papers (0.66) results in a longer decay period (6 to 7 iterations). Hence, while interpreting a scenario dynamics, it can be assumed that an iteration corresponds to a period between 1 and 2 years.

It is difficult to give an ultimate answer, how realistic is the proposed FCM model. It results from a subjective evaluation of causal dependencies between concepts, whereas, FCMs are often created by merging contributions of several experts, who independently identify concepts and propose weight of influences. It should be also mentioned that the model is dedicated to a particular class of academic units in the area of technical sciences. The discussed results should be treated as a forecast, which indicates development directions and makes an attempt to find justifications for them.

The proposed approach can be enhanced by building more accurate FCM models, in particular, by involving in the exercise a broader group of stakeholders and applying voting mechanisms $[4,7]$ to select weights of influences, making further refinements through the decomposition of concepts and the introduction of additional decision variables, e.g. Employees appraisals. 


\section{References}

[1] Axelrod R.M., Structure of Decision: The Cognitive Maps of Political Elites. Princeton University Press, New Jersey, 1976.

[2] Kosko B., Fuzzy Cognitive maps. International Journal of Machine Studies 24, 1986, pp. 65-75.

[3] Kosko B., Neural networks and fuzzy systems: a dynamical systems approach to machine intelligence. Englewood Cliffs USA 449, Prentice Hall, 1992.

[4] Jetter A., Schweinfort W., Building scenarios with Fuzzy Cognitive Maps: An exploratory study of solar energy. Futures 43, 2011, pp. 52-66.

[5] Özesmi U., Özesmi S., Ecological models based on people’s knowledge: A multi-step fuzzy cognitive mapping approach. Ecological Modelling 176, 2004, pp. 43-64.

[6] Aguilar J., A Survey about Fuzzy Cognitive Maps Papers (Invited Paper). International Journal 3, 2005, pp. 27-33.

[7] Papageorgiou E.I., Learning Algorithms for Fuzzy Cognitive Maps - A Review Study. Construction, 2011, pp. 1-14.

[8] Bueno S., Salmeron J., Benchmarking main activation functions in fuzzy cognitive maps. Expert Systems with Applications 36, 2009, pp. 5221-5229. 